\title{
Improved CLAHE Enhancement Technique for Underwater Images
}

\author{
Dr. K. Kanthamma \\ Associate Professor, \\ Dept.of.ECE \\ Bapatla Engineering College, Bapatla, Guntur.
}

\author{
G. Geetha, D. Keerthi \\ D. Uday Kiran, Ch. Vinod Kumar \\ UG Research Fellows, ECE Department, Bapatla \\ Engineering College, Bapatla, Guntur.
}

\begin{abstract}
In recent days, a wide range of research has been going on visual enhancement of underwater images, under images in submarine and military operations to discover the submerged structural designing and sea explorations. But the difficulties are increased by diving in the deep ocean for a long time for underwater images. The factors such as scattering resulting from presence of particles inside the water and blurring effects reduce the quality of images being captured by underwater optic camera. There are several algorithms are introduced to improve the visual quality of deep under water images. Therefore, in this project a novel algorithm White balanced CLAHE is to enhance the visual quality of the underwater images will be implemented andcomparison of data with different conventional enhancement algorithms will be illustrated. And by comparing with the different quality metrics of each algorithm we can conclude which method is best to enhance the underwater images for bothdatabase images and real time images. This can be implemented by using MATLAB software.
\end{abstract}

Keywords:- Underwaterimage, Scattering effects, Image enhancement, Histogram equalisation, CLAHE

\section{INTRODUCTION}

In earth $71 \%$ of its surface is covered with water and aquatic plants. There is more enthusiasm to known that what lies in underwater. Now a days an image of deep waters has a demand to large investigation to extent the underwater for sea floor exploration and navigation. The underwater imaging includes the inspection of seabed exploration and plants. And now the search for wrecks up and to the exploration of natural resources. Underwater images are essentially characterized by their poor visibility because light is exponentially attenuated as it travels in the water and the scenes result poorly contrasted and hazy. Due to above reasons, unmanned remote vehicles are use to sea floor explorations.

\subsection{Historical Development}

Underwater image quality improvement approaches are present a path to magnify the object recognition in underwater areas. A wide research started for the improvement of image visual quality, but a very little amount of work has been carried out in this underwater imaging area. In deep under water, image quality is degraded due to poor illumination conditions that present in underwater. And in deep water the light properties differ compared to air. There are several parameters which decreases the image quality in under deep waters. So in order to eliminate all those effects by using several techniques have been implemented now.

\subsection{Need for pre process}

An initial pre processing is needed for under deep water images, because due to the poor image quality when acquiring the image. Importance of pre processing for under water images [1] are given below:In under deep water images the quality of an image is disturbed due to the light ray properties like absorption of light and scattering effects. Especially the specificity of surroundings such as water torridness, light inequalities and more blue complexion or less influential when vehicles are move in underwater.Image and video captured from deep under waters like unknown rigid scene, the depth of the scene and low light sensitivity due to marine snow.

\section{II.TRADITIONAL TECHNIQUES FOR IMAGE ENHANCEMENT}

There are several techniques which are used frequently for processing the image to improve the visual quality. Some the techniques are:

Histogram Equalization

Contrast stretching

Adaptive Histogram Equalization

\subsection{Histogram Equalization:}

If we creating a histogram that means it provides a better visual representation of data distribution. It can displays a large amount of data and frequencies so we have to apply the histogram to improve the image quality.It can display data information that uses rectangles to show the frequency of data information in successive numerical intervals of equal size.

Histogram equalization is a method for adjusting image intensities to enhance image contrast. For a perfect image it has equal number of pixels in all its grey levels [2].It assigns the intensity values of pixels in the input image so that the output image contains a uniform distribution of intensities. This improves contrast and gives a uniform histogram.

By using, histogram it makes our task easier to identify different data. It helps to visualize the distribution of data. But it may increase the contrast of background noise, while decrease the original signal. So further we go for contrast stretching method. 


\subsection{Contrast Stretching:}

The contrast stretching is a method to make brighter portion more brighter and darker portion more darkerby using predefined tansformation function [2]. we have 256 grey levels. ' 0 ' indicates black and white indicates ' 255 '. Usually, Underwater images will have less gray values. So in this method the current grey value of the image is stretched towards 255 i.e.,from black to white and performed pixel by pixel. It means that contrast of the image is improved for better vision.

For example, Take two different threshold values are considered for the entire image and the values between them are stretched to maximum extent, then the contrast of the image is increases. Moreover by this method entire global image contrast is enhanced.

In this method there is drawback that is, the transformation function is not unique. Based on the application the suitable transformation function is to be chosen. So to overcome this problem we go for adaptive histogram equalization.

\subsection{Adaptive Histogram Equalization:}

Adaptive histogram equalization is used to improve the quality and contrast of an image. It is PC based image processing method. And it is similar to the contrast stretching method but with a slight difference.It has several intensities of specific grey value each belongs to a distinct portion of an image, by applying a suitable transformation function intensities are rearranged. Each pixel transformed based on the histogram of a square surrounding the pixel [3].Transformation function is applied on the histogram proportional to the cumulative distributive function (CDF) of pixel values in the neighbourhood. It suits for enhancing the local details and edge information of each region in an image. It is a effective technique which will benefit of the images with extreme contrast values. In this, the limitation is unwanted noise present in the background of an image and it leads to loss in information signal [4]. The noise in homogeneous regions of an image are amplified it results in poor SNR. Only the local objects of the image are enhanced but the background is unenhanced.

\section{PROPOSED METHOD}

\subsection{White BalancedContrast Limited Adaptive Histogram Equalization:}

Before we apply this method we have to balance the photometric variations by using gray world approach or white balanced approach.

\subsection{Balancing of Photometric variations:}

To acquire an image a source of illumination is required. In deep under water the main source of illumination is sun. The acquired image is RGB type, in deep underwater blue colour variations are more dominant due to its shorter wavelength. Usually colour images consist of 3 planes i.e., red, green, blue due to improper illumination source impact of blue colour compared to green and red is more. Due to this effect there is a necessity to balance the colour combination so it is invariant of illumination.

So in order to balance those variations, assess the colour of predominating light and remove effects of that colour to make the acquired image free from photometric variations. These effects are removed by transformation on pixel intensities of each colour plane by an average pixel intensity value of that colour plane [5].

\subsection{White Balanced Approach:}

This method is also known as Gray World Approach. In this, colour constancy is a process of recognition of colour independent of light. Illuminants is the cause of colour casts in captured digital image. To solve this colour constancy problem first it has to estimate colour of prevailing light and then it remove the unwanted colour casts. Gray World(GW) techniqueis widely used and general white balance algorithm. This algorithm produces an estimate of illumination by calculating the mean of each channel of the image [6].The average of each individual channel is used to calculate a separate scaling value for every channel. By this way the illumination on different channel is eliminated independently. when we using this method it is important to keep the following. An image in which many similar colours are present gives a bad result because this method needs a wide range of colours. This algorithm illuminates this dominant colour. This function can be executed on every image represented by both in one dimensional and three dimensional matrix.

\subsection{Contrast Limited adaptive histogram equalization:}

Ordinary AHE tends to overamplify the contrast in near constant regions of the image.It is originally developed for enhancement of low contrast images [7].CLAHE is a variant of adaptive histogram equalisation in which contrast amplification is limited,so as to reduce this problem of noise amplification.In order to limit the noise amplification we use CLAHE [8].In CLAHE,the contrast limited procedure has to be applied for each neighbourhood from which a transformation function is derived.Rather than taking the whole image, CLAHE prevents over amplification by dividing the image into small data regions called tiles and then it performs contrast enhancement. And these tiles are rejoined to get an overall enhanced image. It is applied over both type of images gray scale and coloured.

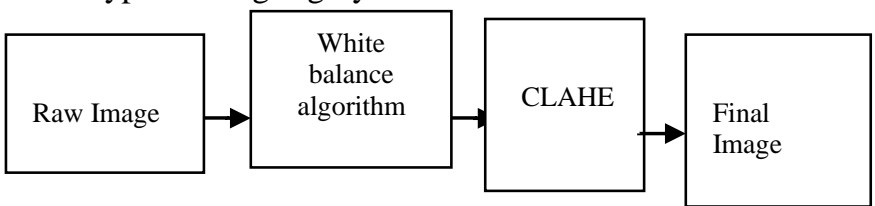

Fig 1. Block diagram of White balanced CLAHE

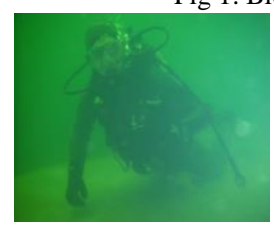

(a)

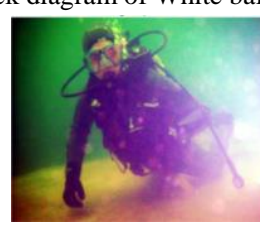

(b)

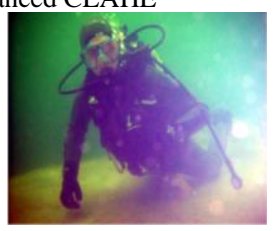

(c) 


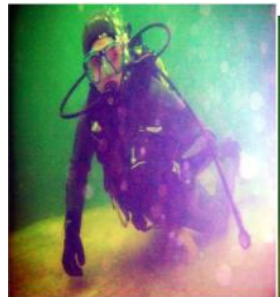

(d)

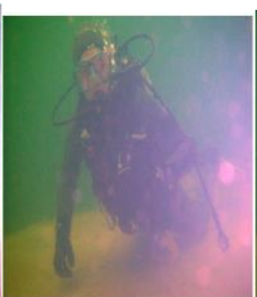

(e)

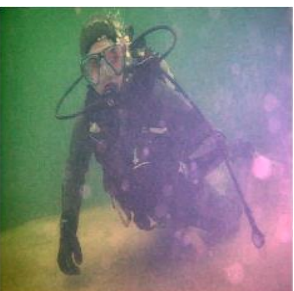

(f)
Fig.2 (a) Original image, (b) HE, (c) Contrast stretching, (d) AHE (e) White balance, (f) White balanced CLAHE

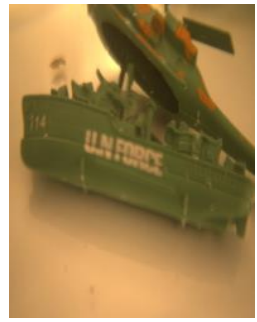

(a)

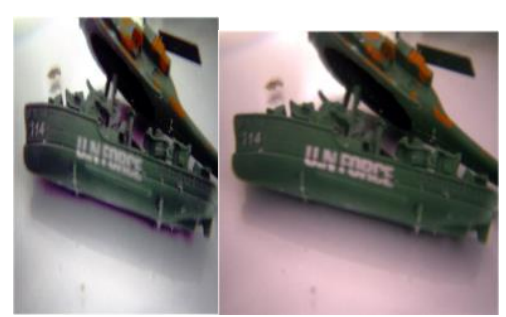

(c)

(b)

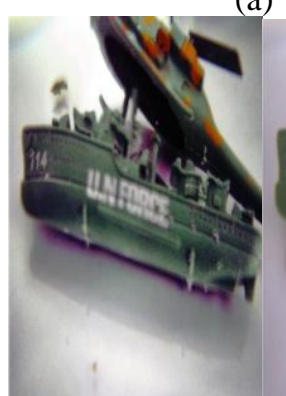

(d)

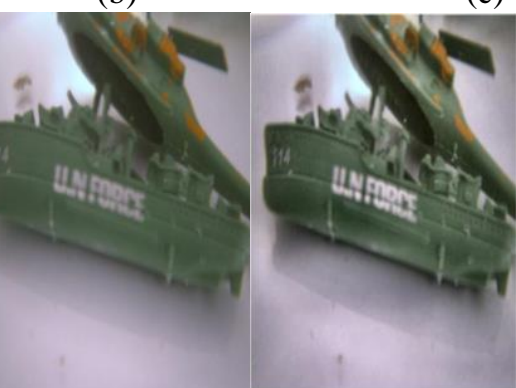

(e) (f)
Fig.2 (a) Original image, (b) HE, (c) Contrast stretching, (d) AHE (e) White balance, (f) White balanced CLAHE

Table:1 Comparison of Traditional and White balanced CLAHE techniques(Fig1)

\begin{tabular}{|l|l|l|l|l|}
\hline $\begin{array}{l}\text { Quality } \\
\text { Metrics }\end{array}$ & $\begin{array}{l}\text { Histogram } \\
\text { Equalization }\end{array}$ & $\begin{array}{l}\text { Contrast } \\
\text { Stretching }\end{array}$ & $\begin{array}{l}\text { Adaptive } \\
\text { Histogram } \\
\text { Equalization }\end{array}$ & $\begin{array}{l}\text { White } \\
\text { Balanced } \\
\text { CLAHE }\end{array}$ \\
\hline PSNR(dB) & 54.8446 & 54.8811 & 54.8610 & 55.5618 \\
\hline MSE & 0.2143 & 0.2130 & 0.2140 & 0.1821 \\
\hline RMSE & 0.4629 & 0.4615 & 0.4626 & 0.4267 \\
\hline
\end{tabular}

Table:2 Comparison of Traditional and White balanced CLAHE techniques(Fig2)

\begin{tabular}{|c|c|c|c|c|}
\hline $\begin{array}{c}\text { Quality } \\
\text { Metrics }\end{array}$ & $\begin{array}{c}\text { Histogram } \\
\text { Equalization }\end{array}$ & $\begin{array}{c}\text { Contrast } \\
\text { Stretching }\end{array}$ & $\begin{array}{c}\text { Adaptive } \\
\text { Histogram } \\
\text { Equalization }\end{array}$ & $\begin{array}{c}\text { White } \\
\text { Balanced } \\
\text { CLAHE }\end{array}$ \\
\hline PSNR(dB) & 54.8120 & 54.8372 & 54.8216 & 55.3462 \\
\hline MSE & 0.2149 & 0.2152 & 0.2159 & 0.1914 \\
\hline RMSE & 0.4628 & 0.4639 & 0.4667 & 0.4375 \\
\hline
\end{tabular}

\section{CONCLUSION}

In this paper, different underwater image enhancement techniques are studied and in the field of image processing, there are several techniques are proposed to enhance the visual quality of underwater images. Existing techniques improves the deep water images to a large extent. But all these techniques suffer with poor quality. For a good quality image it will have high PSNR and low MSE. Based on these characteristics the proposed method, and the conventional method is compared. The results that have been obtained for both the techniques, White Balanced CLAHE gives the better enhancement of quality parameters for underwater images.

\section{REFERENCES}

[1] Padmavathi, G., et al. "Comparison of filters used for underwater image preprocessing." International Journal of Computer Science and Network Security10.1 (2010): 58-65.

[2] Balvant singh, Ravi shankar mishra, Puran gour, "Analysis of contrast enhancement techniques for underwater image" International journal of computer technology and electronics engineering (IJCTEE) Volume 1, issue 2, pp: 190-195.

[3] Ritu singh, Dr. Mantosh Biswas, "Adaptive histogram equalization based fusion Technique for hazy underwater image enhancement" IEEE international conference on computational intelligence and computing research, 2016, pp: 1-5.

[4] Sonam Bharal, "Review of underwater image enhancemen techniques", International Research Journal of Engineering and Technology (IRJET), Volume: 02 Issue: 03, June-2015, pp: 340-344.

[5] Ch. Jaya Lakshmi, K. Prasanti, S. Kalpana "Visual Enhancement Techniques For Underwater Images" 5 th International Conference on Advanced Computing \& Communication Systems (ICACCS), 2019, pp: $114-117$

[6] Sudhansu Mallik, Salman S. Khan, Umesh C. Pati, "Visual Enhancement of Underwater Image by White-Balanced EMD" 8th International Conference on Computing, Communication and Networking Technologies (ICCCNT) -2017, pp: 1-6.

[7] Muhammad Suzuri Hitam , Ezmahamrul Afreen Awalludin , Wan Nural Jawahir Hj Wan Yussof, Zainuddin Bachok "Mixture contrast limited adaptive histogram equalization for underwater image enhancement" International Conference on Computer Applications Technology (ICCAT), 2013

[8] Lintao Zheng, Hengliang Shi, Shibao Sun "Underwater Image Enhancement Algorithm Based on CLAHE

and USM" IEEE International Conference on Information and Automation(ICIA),2016, pp:585-590. 